\title{
Reversing type 2 diabetes: journal review and a case study
}

\begin{abstract}
Type 2 diabetes (T2D) has for quite some time been recognized as a serious interminable malady dependent on conventional methods for treatment. Exploration currently exists that proposes reversal is conceivable through different implies that have as of late been grasped in the rules. This account audit looks at the proof for T2D reversal utilizing every one of the three strategies, including points of interest and constraints for each. Techniques: A research was performed, and an aggregate of unique articles containing data relating to diabetes reversal or abatement were incorporated. Results: Evidence exists that T2D reversal is attainable utilizing bariatric medical procedure, low-calorie nutrient dense and moderate protein diet (LCNP), or starch limitation (LC). Bariatric medical procedure has been suggested for the therapy of T2D since 2016 by a global diabetes agreement gathering. Both the American Diabetes Association (ADA) and the European Association for the Study of Diabetes (EASD) presently suggest a LC eating example and backing the momentary utilization of LCNP for weight reduction. Nonetheless, just T2D treatment, not reversal, is talked about in their rules. End: Given the condition of proof for T2D reversal, medical services suppliers should be taught on reversal choices so they can effectively take part in guiding patients who may want this way to deal with their sickness.
\end{abstract}

Keywords: low calorie, diabetes, diabetes reversal, bariatric surgery, very-low-calorie, low-carbohydrate
Volume 10 Issue 5 - 2020

\section{Ruchi Singh}

Health counselor \& Nutritionist, Long Island University, USA

Correspondence: Ruchi Singh, Health counselor \&

Nutritionist, Long Island University, USA,

Email ruchiaug@gmail.com

Received: September 15, 2020 | Published: October 30, 2020
Abbreviations: LC, starch limitation; LCNP, low-calorie consumes less calories; T2D, type 2 diabetes

\section{Introduction}

India is one of the 7 nations of the IDF SEA locale. 463 million individuals have diabetes on the planet and 88 million individuals in the SEA Region; by 2045 this will ascend to 153 million (South East Asia). Diabetes is a significant general wellbeing pestilence in spite of late advances in both drug and technologic treatment alternatives. Type 2 diabetes (T2D) has for some time been recognized as a hopeless incessant infection. The best result that has been normal is enhancement of diabetes side effects or easing back its unavoidable movement. Roughly half of T2D patients will require insulin treatment inside ten years of determination Although in the past diabetes has been called constant and irreversible, the worldview is changing.

As indicated by World Health Organization (WHO) ${ }^{1}$ Diabetes is a ceaseless, metabolic infection portrayed by raised degrees of blood glucose (or glucose), which leads after some time to genuine harm to the heart, veins, eyes, kidneys and nerves (World Health Organization). The most well-known is type 2 diabetes, generally in grown-ups, which happens when the body gets resistace to insulin or doesn't make enough insulin. In the previous thirty years the pervasiveness of type 2 diabetes has risen significantly in nations of all pay levels. Type 1 diabetes, when known as adolescent diabetes or insulin-subordinate diabetes, is an interminable condition in which the pancreas delivers practically no insulin without anyone else. For individuals living with diabetes, admittance to moderate treatment, including insulin, is basic to their endurance. There is a worldwide concurred focus to stop the incidence in diabetes and weight by 2025 .

Around 422 million people worldwide have diabetes, and 1.6 million passings are direct credited to diabetes consistently. Both the amount of cases and the transcendence of diabetes have been reliably growing over the span of late decades. (WHO) "Diabetes reversal" is a term that has discovered its way into logical articles and the lay press the same; "reduction" has additionally been utilized. While the specific models are still discussed, most concur that a hemoglobin A1c (HbA1c) under the diabetes limit of $6.5 \%$ for an all-inclusive timeframe without the utilization of glycemic control drugs would qualify. Barring metformin from the glycemic control prescriptions list, as it has signs past diabetes, may likewise be a thought (\#B8supplements 11-00766, 2019). Moreover, terms, for example, "fractional" (HbAlc $<6.5$ without glycemic control drugs for 1 year) or "complete" (HbA1c <5.7 without glycemic control prescriptions for 1 year) abatement have been characterized by a specialist board as more proof amasses that focuses to the chance of staying away from the apparently dynamic nature of T2D. Note that the expression "fix" has not been applied to T2D, as there exists the potential for reoccurrence, which has been very much archived in the literature. Notwithstanding the developing proof that reversal or reversal is conceivable, accomplishing reversal isn't generally energized or encouraged by our medical care framework. Indeed, reversal isn't an objective in diabetes rules. Explicit intercessions focused on reversal all make them thing in like manner: they are not first-line standard of care. This is significant; on the grounds that there is proof proposing that norm of care doesn't prompt diabetes reversal. This brings up the issue of whether standard of care is actually the best practice. Many found a diabetes abatement and the norm approach won't opposite the wellbeing emergency of diabetes.

A noteworthy number of studies demonstrate that diabetes reversal is reachable utilizing bariatric medical procedure, while different methodologies, for example, low-calorie consumes less calories (LCNP), have likewise indicated adequacy in an expanding number of studies. This case study will review and illustrate the important aspects 
of significant improvement with dietary approaches, identifying their beneficial effects, supporting evidence, drawbacks, and degree of sustainability.

\section{Material and methods}

A writing search was preceded as proper for account surveys, including electronic information bases of PubMed, EMBASE, and Google Scholar from 1970 through December 2018. I inspected English-language unique and survey articles found under the subject headings diabetes, bariatric medical procedure, metabolic medical procedure, low-calorie diet, calorie limitation, low starch diet, ketogenic diet, diabetes abatement, diabetes reversal and actualized the low calories, complex sugars, moderate to high protein and supplement thick eating regimen. References of the distinguished distributions were looked for more examination articles to remember for this survey. Chosen contemplates were assessed and assessed for qualification for incorporation in this audit dependent on their pertinence for diabetes reversal and reduction. Either abatement or reversal should have been examined in the paper or the outcomes were reliable with these terms for consideration. Randomized clinical preliminaries and intercession based examinations were given accentuation for consideration.

\section{Bariatric surgery}

Bariatric medical procedure has for quite some time been perceived as an expected therapy for both morbid obesity and the metabolic cycles that go with it, explicitly T2D. While the viability of T2D reversal relies upon the decision of method, there is one-sided improvement in glycemia following activity, and bariatric medical procedure has been discovered to be better than concentrated T2D clinical administration. Likewise, in 2016, the second Diabetes Surgery Summit (DSS-II) delivered proposals, embraced by 45 clinical and logical social orders around the world, to utilize bariatric medical procedure as a therapy for T2D (bariatric medical procedure is as of now affirmed by the 2016 suggestions for grown-ups with a weight list (BMI) $>40$, or $>35$ $\mathrm{kg} / \mathrm{m}^{2}$ with heftiness related comorbidities). ${ }^{2}$ Of intrigue is the reliable finding that glycemic enhancements happen quickly, regularly inside hours to days, and go before weight reduction, which probably speaks to the enteroendocrine reactions to adjusted progression of intestinal substance (i.e., bile corrosive flagging and changes in microbiota and their metabolome). Besides, microbial changes in the human gut have been connected to stoutness, and careful adjustments to gastrointestinal life systems have been related with emotional changes in gut microbiota population with reversal from an "obesogenic" to a lean bacterial populace. ${ }^{3}$

Long haul results from bariatric medical procedure rely upon numerous variables, including sort of medical procedure performed, understanding comorbidities, persistent preparation for deep rooted dietary change, and continuous reconnaissance. While bariatric medical procedure has been exhibited to be protected and compelling generally, perceive that it isn't without hazards. Every patient must gauge the dangers and advantages related with untreated bleak stoutness versus those related with medical procedure or powerful dietary administration and pick appropriately. Critical inconveniences incorporate drain, post-usable readmission, requirement for reoperation, post-employable hypoglycemia, dumping disorder, declining indigestion, peripheral ulceration, and micronutrient lacks.

It is critical to consider that while brief length contemplates have indicated early goal of comorbidities following bariatric techniques, when followed for various decades, there might be diminished adequacy of sickness goal and expanded rate of emergency clinic confirmation long haul. Long haul reversal of T2D and genuine glucose homeostasis stay dubious. Weight reduction after medical procedure is a critical indicator of a re-visitation of euglycemia ${ }^{4}$ post-operatively. Various investigations have detailed beginning T2D abatement rates as high as $80 \%$, notwithstanding, long haul reduction is less sturdy.

\section{Low-calorie diets, complex carbohydrates, nutrient dense and moderate quality protein diet (LCNP)}

As diabetes rates have risen to unprecedented levels (Centers for Disease Control and Prevention), the number of studies examining diabetes reversal using non-surgical techniques has increased. A handful of studies have reported successful weight loss with decreased insulin resistance, plasma glucose, and medication use following a LCNP.Mrs. Vermahas been gaining weight over the last five years and her $\mathrm{HbA} 1 \mathrm{c}$ is keep on increasing anddoctor has said that she needs to be careful with the diet and exercise more andsuggested time releasemetformin 500 gm twice a day. Mrs. Verma is 49 , a middle age weighing around 70 kegs', who really doesn't want to follow any diet regime and do any exercise for her health as she is too busy to do anything extra for herself.After four to five times of counseling and small interventions of dietary modification and exercise implementation, she started losing fat while preserving the muscle mass and gaining confidence. After identifying the nutrients gaps and deficiencies with extensive counseling of 6 months of dietary recommendations were given along with herbs and vitamin and omega 3 supplementation and after 6 monthsHbAlc improved from 6.9 to 5.9 Other significant improvement were notices in energy level, low water retention and lean muscle to fat ratio. She was much more active than currently and would go for walks in Sunrise Park by herself when the weather was good and she seemed to enjoy that.Now her dosages of diabetes medicine were reduced to half.

In 1976, Bistrian et al., 5 revealed that a low-calorie protein-saving changed quick took into account insulin end in every one of the seven corpulent patients with T2D. An examination by Bauman et al., a lowcalorie diet of $900 \mathrm{kcal}$, including $115 \mathrm{~g}$ of protein, prompted huge improvement in glycemic control that was for the most part ascribed to upgrades in insulin affectability. ${ }^{6}$ Besides, an examination led in corpulent T2D patients found that a LCNP and gastric detour medical procedure were similarly compelling in accomplishing weight reduction and improving glucose and $\mathrm{HbAlc}$ levels in the present moment. ${ }^{7}$ Weight reduction, notwithstanding, endured in the eating routine treated patients just for the initial three months, showing trouble with long haul upkeep. ${ }^{8}$ Likewise, different examinations additionally revealed comparative example of early blood glucose standardization without medicine use, however the enhancements were not supported long haul. ${ }^{9}$ Moreover, the examination by Wing et al., ${ }^{10}$ despite the fact that revealed huge and more noteworthy upgrades of $\mathrm{HbAlc}$ at 1 year in the discontinuously conveyed exceptionally low-calorie diet, the HbA1c improvement was not fundamentally unique in relation to what was accounted for in the patients getting low-calorie diet (LCNP) all through the one year time frame. Besides, the glycemic enhancements saw at 1 year were not kept up through 2-years, despite the fact that the gathering with discontinuous lowcalorie diet had less drug prerequisite than the gathering in the LCNP arm at 2 years. In conclusion, micronutrient insufficiencies with the utilization of calorie confined weight control plans has been appeared and supplementation and observing for inadequacies is a thought with their utilization. ${ }^{11}$ 
While these past investigations were not surveying diabetes reduction or reversal rate as such, they exhibited the adequacy of calorie limitation in accomplishing weight reduction and improved glycemic control, which are the center objectives of reversal. In 2003, the Look AHEAD preliminary randomized 5145 overweight or fat patients with T2D to an intercession bunch that got either a serious way of life mediation (ILI) including calorie limitation and expanded physical movement or to a benchmark group that included diabetes backing and instruction (DSE).$^{12}$ Post hoc investigation of this examination uncovered that at one year, $11.5 \%$ of the members in the ILI bunch accomplished reduction (incomplete or complete); nonetheless, abatement rates thusly diminished after some time $(9.2 \%$ at year two and $7.3 \%$ at year four). In any case, the reduction rates accomplished through ILI were three to multiple times higher than those accomplished in the DSE gathering. Lower gauge $\mathrm{HbA} 1 \mathrm{c}$, more noteworthy degree of weight reduction, shorter length of T2D conclusion, and absence of insulin use at pattern anticipated higher abatement rate in ILI members. ${ }^{13}$

An underlying 2011 diabetes reversal concentrate by Taylor and partners demonstrated that a low-calorie diet of $600 \mathrm{Kcal} /$ day not just standardized glucose, $\mathrm{HbA1c}$, and hepatic insulin affectability levels inside seven days, yet additionally prompted diminished hepatic and pancreatic triacylglycerol substance and standardization of the insulin reaction inside about two months. ${ }^{14}$ At 12 weeks post-mediation, a considerable lot of the upgrades were kept up, yet over a fourth of the patients had an early repeat of diabetes. Taken together, proof recommends that a LCNP is successful in switching diabetes in the present moment as long as two years, and its adequacy was dominatingly shown in those with shorter length since diabetes determination. Note that a considerable degree of calorie limitation is expected to create an adequate degree of weight reduction for turning around diabetes. Transient intercession with moderate vitality limitation and metformin for unobtrusive weight reduction was not as compelling in turning around diabetes when contrasted with standard diabetes care. ${ }^{15}$ Way of life mediation with extreme vitality limitation may have some harmful impact on the body arrangement and physiology, which represents a worry for long haul wellbeing. Moreover, long haul accomplishment of diabetes reduction, adherence to the eating routine, and weight reduction upkeep after the eating regimen stay a test. Studies have likewise proposed that physiological and metabolic transformation of the body because of caloric limitation may move vitality balance and hormonal guideline of weight toward weight recapture after weight reduction. ${ }^{16}$ Subsequently, it is significant that future examinations are coordinated towards evaluating the drawn out sustainability of diabetes abatement drove by LCNP and feasibility of this eating routine on the physiological variation and body arrangement changes.

\section{Carbohydrate-restricted diets (LC)}

Prior to the disclosure of insulin in 1921, low starch (LC) consumes less calories were the most habitually endorsed treatment for diabetes. ${ }^{17}$ The outlook changed both with the advancement of exogenous insulin and later with the rise of the low-fat eating routine worldview. An eating routine low in fat, which as a matter of course is high in starch, turned into the standard proposal in rules the world over. ${ }^{18}$ As opposed to forestalling heights in glucose, the objective became support of glucose control by means of the expanded utilization of glycemic control meds, including insulin..$^{19}$ In the course of the most recent decade, clinical examinations have started to revive the pre-insulin LC dietary methodology. Because of the new proof on the adequacy of sugar limitation, low-starch has as of late been supported as an eating design by the ADA and the European Association for the Study of Diabetes (EASD) ${ }^{20}$ Furthermore, the Veterans Affairs/Department of Defense (VA/DOD) rules presently suggest starch limitation as low as $14 \%$ of vitality admission in its latest rules for treatment of diabetes (VA) (Department of Veteran Affairs and Department of Defense VA/DoD Clinical Practice Guideline for the Management of Type 2 Diabetes Mellitus in Primary Care. Version 5.0). ${ }^{21}$

LC consumes less calories depend on macronutrient changes instead of an emphasis on calorie limitation..$^{22}$ Despite the fact that the specific definition differs, a low-sugar diet generally limits absolute starches to under 130 grams for each day, while a low-sugar or ketogenic diet as a rule confines complete starches to as low as 20-30 grams for every day. Protein utilization is commonly unaltered from a standard ADA diet (around 20\% of admission), with the rest of the vitality needs met by fat from either the eating regimen or mobilized muscle to fat ratio stores. Sugar sources are principally non-bland vegetables with certain nuts, dairy, and restricted organic product.

A sum of 32 separate preliminaries inspecting starch limitation as a treatment for T2D were discovered when our pursuit was performed. Nonetheless, for reasons that may incorporate changed degrees of starch limitation and varying degrees of help given, not all investigations had results that would be reliable with diabetes reversal. Various shorter-term preliminaries have discovered a huge between-bunch favorable position of a low-starch mediation for T2D. ${ }^{23}$ Information from longer-term preliminaries are restricted, and in some subsequent investigations, the between-bunch advantage seen at first was lost or decreased, in spite of the fact that it regularly remains essentially improved from standard. This brings up the issue of long haul sustainability utilizing this methodology. Because of heterogeneity in philosophy and meaning of sugar limitation, the ability to completely look at T2D reversal dependent on the current investigations is restricted. In light of an ongoing deliberate audit of LC, apparently the best upgrades in glycemic control and most noteworthy prescription decreases have been related with the least starch admission. ${ }^{24}$ With regards to these constraints, it seems critical to survey the degree of starch limitation, uphold or different strategies given to energize sustainability, and length of development.

An assessment differentiating a not mandatory low-starch $(<20$ g outright) diet to an imperativeness restricted low-glycemic diet in T2D found more conspicuous abatement in $\mathrm{HbA} 1 \mathrm{c}$, weight, and insulin levels in the low-sugar arm. Additionally, $95 \%$ of individuals in the low-sugar arm diminished or cleared out glycemic control drugs, appeared differently in relation to $62 \%$ in the low glycemic record arm at 24 weeks. Direction was given in a one-time meeting with a dietician and included bring home materials for reference. A to some degree longer examination (34 weeks) $\operatorname{primer}^{25}$ found that an extraordinarily low-sugar ketogenic diet intercession (20-50 $\mathrm{g}$ net carbs consistently) occurred in HbA1c underneath the cutoff for diabetes in $55 \%$ of the patients, diverged from $0 \%$ of patients in the low-fat arm. The preparation gatherings were all on the web and included lead change frameworks and cautious eating which was relied upon to address glutting. New activities were informed to the patients step by step for the underlying four months and a while later at ordinary spans for the remainder of the assessment.

A little (34 members) one-year investigation of a not obligatory, low-sugar diet contrasted with a calorie-limited moderate starch diet found a critical decrease in $\mathrm{HbA} 1 \mathrm{c}$ between bunches preferring the lowsugar arm. ${ }^{25}$ At one year, $78 \%$ of members who started the preliminary 
with a $\mathrm{HbAlc}$ above $6.5 \%$ no longer met the cutoff for the finding of diabetes, not, at this point required any non-metformin drug, and essentially decreased or disposed of metformin. Complete kilocalorie admission was not fundamentally extraordinary between the two gatherings, even with moderate sugar limitation. Notwithstanding equivalent vitality consumption, the low sugar bunch lost altogether more weight and had improved glycemic control, which shows an expected unthinking part for starch limitation itself. The help given was 19 classes over the year time frame, tightening in recurrence after some time.

Another year preliminary ${ }^{26}$ discovered noteworthy $\mathrm{HbA} 1 \mathrm{c}$ decrease in the subset of patients with diabetes $(n=54)$ allocated to a not indispensable low-starch diet $(<30$ absolute grams every day), contrasted with a vitality limited low-fat eating routine. These outcomes stayed noteworthy subsequent to modifying the model for weight reduction, showing an impact of the starch decrease itself. The help given was four week after week meetings during the main month, trailed by month to month meetings for the staying 11 months.

\section{Summary}

Many evidences showed up as of late recommending that diabetes reversal is a potential choice to consider instead of conventional diabetes treatment and the executives. In this paper, we give an audit of three techniques that have been appeared to effectively invert type 2 diabetes. The current group of proof recommends that bariatric medical procedure is the best technique for in general adequacy and delayed abatement, despite the fact that worries related with careful complexities, therapy cost and complete way of life change after medical procedure remain difficulties for wide reception of this methodology. While both the LCNP and LC dietary methodologies are persuading for switching diabetes temporarily (as long as two years), long haul upkeep of diabetes abatement is as yet problematic. There are restricted accessible information supporting long haul upkeep of weight reduction and its related glycemic enhancements in light of LCNP; also, long haul adherence to a low sugar diet will probably stay an obstruction without the improvement of legitimate patient instruction and ideal help for long haul conduct change. Besides, research in understanding the instrument of diabetes reversibility in each of the three methodologies and its covering unthinking pathways are inadequate with regards to; this is a territory for future exploration accentuation.

There are comparable recognized negative indicators of abatement for each of the three methodologies. These variables incorporate longer diabetes length and expanded seriousness, lower BMI, progressed age, poor glycemic control, and low C-peptide levels (demonstrating diminished endogenous insulin creation). ${ }^{27}$ Further investigation into the heterogeneity of these elements will help customize the methodology, decide sensible objectives for every patient, and ought to be considered during treatment conversations. Progressing examination into calculation advancement will be useful in such manner. ${ }^{29-33}$

\section{Conclusion}

In summary, as a general public we cannot manage the cost of anymore or endure the kept increasing paces of diabetes. In spite of numerous boundaries inside the medical services framework overall, providers are capable consistently for the lives of patients got up to speed in this exceptional pestilence. The current norm of care might be appropriate for a few, however others would without a doubt pick reversal on the off chance that they comprehended there was a decision. The decision must be offered if providers are mindful that reversal is conceivable as well as have the training expected to audit these choices in a patient-driven conversation.

\section{Acknowledgments}

None.

\section{Conflicts of interest}

The author declares that there are no conflicts of interest.

\section{Funding}

None.

\section{References}

1. Global report on diabetes.

2. Francesco Rubino, David M Nathan, Robert H Eckel, et al. Metabolic surgery in the treatment algorithm for type 2 diabetes: A joint s. International Diabetes Organizations. Obes Surg. 2017;27(1):2-21.

3. Sleeve DC. Gastrectomy Bariatric Surgery Vary According to Diabetes Remission. 2017.

4. Federico A, DM. Gastrointestinal Hormones, Intestinal Microbiota and Metabolic Homeostasis in Obese Patients: Effect of Bariatric Surgery: Effect of Bariatric Surgery. 2016;321.

5. B R Bistrian, G L Blackburn, J P Flatt, et al. Nitrogen metabolism and insulin requirements in obese diabetic adults on a protein-sparing modified fast. Diabetes. 1976;25(6):494-504.

6. Bauman WA. Early and long-term effects of acute caloric deprivation in obese diabetic patients. The American Journal of Medicine. 1988;85(1):38-46.

7. Hughes TA, GJ. Effects of caloric restriction and weight loss on glycemic control, insulin release and resistance, and atherosclerotic risk in obese patients with type II diabetes mellitus. Am J Med. 1984;77:7-17.

8. Lars Sjöström, Anna-Karin Lindroos, Markku Peltonen, et al. Lifestyle, diabetes and cardiovascular risk factors 10 years after bariatric surgery. $N$ Engl J Med. 2004;351(26):2683-2693.

9. Hammer S, SM. Prolonged caloric restriction in obese patients with type 2 diabetes mellitus decreases myocardial triglyceride content and improves myocardial function. Journal of the American College of Cardiology. 2008;1006-1012.

10. R R Wing, E Blair, M Marcus, L H Epstein, et al. Year-long weight loss treatment for obese patients with type II diabetes: Does including an intermittent very-low-calorie diet improve outcome? J Med. 1994;354362 .

11. Antje Damms-Machado, Gesine Weser, Stephan C Bischoff. Micronutrient deficiency in obese subjects undergoing low calorie diet. Nutr J. 2012;11:34.

12. Donna H Ryan, Mark A Espeland, Gary D Foster, et al. Look AHEAD Research Group Look AHEAD (Action for Health in Diabetes): Design and methods for a clinical trial of weight loss for the prevention of cardiovascular disease in type 2 diabetes. Control Clin Trials. 2003;24(5):610-628.

13. Edward W Gregg, Haiying Chen, Lynne E Wagenknecht, et al. Association of an intensive lifestyle intervention with remission of type 2 diabetes. JAMA. 2012;2489-2496.

14. EL Lim , K G Hollingsworth, B S Aribisala, et al. Reversal of type 2 diabetes: Normalisation of beta cell function in association with decreased pancreas and liver triacylglycero. Diabetol. 2011;2506-2514. 
15. McInnes N, SA. Piloting a remission strategy in type 2 diabetes: Results of a randomized controlled trial. J Clinical Endbrinolgy. 2017;1596-1605.

16. Erin Fothergill, Juen Guo, Lilian Howard, et al. metabolic adaptation 6 years after. The Biggest Loser" competition. Obesity. 2016;1612-1619.

17. W R Campbell. Dietetic treatment in diabetes mellitus. Medical Association Journal. 1923;487-492.

18. R Arky, J Wylie-Rosett, B El-Beheri. Examination of current dietary recommendations for individuals with diabetes mellitus. Diabetes care. 1982;5(1):59-63.

19. Anderson JW. New perspectives in nutrition management of diabetes mellitus. American Medical Journal. 1988;159-165.

20. American Diabetes Association Summary of Revisions: Standards of Medical Care in Diabetes.

21. Department of Veteran Affairs and Department of Defense. VA/DoD Clinical Practice Guidelines for the Management of Type 2 Diabetes Mellitus in Primary Care. Version 5.0. 2020.

22. Eric C Westman, Richard D Feinman, John C Mavropoulos, et al. Low carbohydrate nutrition and metabolism. Journal Clinical Nutrition. 2007;276-284.

23. Daly ME PR. Short-term effects of severe dietary carbohydrate-restriction advice in type 2 diabetes: A randomized controlled trial. Diabet. Medical Journal. 2006;15-20.

24. Ole Snorgaard, Grith M Poulsen, Henning K Andersen. Systematic review and meta-analysis of dietary carbohydrate restriction in patients with type 2 diabetes. BMJ Open Diabetes Res Care. 2017;5(1):e000354.
25. Laura R Saslow, Jennifer J Daubenmier, Judith T Moskowitz. Twelvemonth outcomes of a randomized trial of a moderate-carbohydrate versus very low-carbohydrate diet in in overweight adults with type 2 diabetes mellitus or prediabetes. Nutr Diabetes. 2017;7(12):304.

26. Stern L, IN. The effects of low-carbohydrate versus conventional weight loss diets in severely obese adults: One-year follow-up of a randomized tria. Internal Medicine. 2004;778-785.

27. Min T, BJ. Predicting the Resolution of Type 2 Diabetes after Bariatric Surgical Procedures: A Concise Review. J Diabetes Metabolism. 2015;6617.

28. Centers for Disease Control and Prevention. from Centers for Disease Control and Prevention. 2020.

29. Reversing Type 2 Diabetes: A Narrative Review of the Evidence. Nutrients. 2019;11(4):766.

30. Walter J Pories, James H Mehaffey, Kyle M Staton. The surgical treatment of type two diabetes mellitus. Surg Clin North Am. 2011;91(4):821-836.

31. IDF Diabetes Atlas: Global estimates of the prevalence of diabetes for 2011 and 2030. 2011.

32. IDF SEA members.

33. Diabetes. 